\title{
Degenerações periféricas da retina do olho míope X LASIK
}

\author{
Peripheral retinal degenerations in myopic eye X LASIK
}

João J. Nassaralla Jr. ${ }^{1}$

Belquiz A. Nassaralla ${ }^{2}$

\section{RESUMO}

O objetivo deste artigo é apresentar as degenerações periféricas mais comuns na retina dos olhos míopes, discutindo aquelas que oferecem maiores riscos para o descolamento regmatogênico da retina, seu relacionamento com a cirurgia de LASIK e a indicação para o tratamento profilático.

Descritores: Degeneração retiniana/prevenção \& controle; Miopia/complicações; Ceratomileuse assistida por excimer laser in situ

\section{INTRODUÇÃO}

A miopia é uma das maiores causas de cegueira legal em todo o mundo. O déficit visual ocorre em idade precoce, comparado à faixa etária das outras quatro principais causas: catarata, retinopatia diabética, glaucoma e degeneração macular relacionada à idade, que ocorrem décadas depois. Do ponto de vista social, a miopia causa um enorme impacto, pois pode alterar o desempenho de indivíduos na etapa produtiva de suas vidas ${ }^{(1)}$.

Entre as causas de cegueira associadas à miopia predominam as alterações do segmento posterior do bulbo ocular, tais como: descolamento regmatogênico da retina (DR), alterações degenerativas maculares, estafilomas posteriores com ectasia macular, neovascularização sub-retiniana macular, buraco de mácula e glaucoma ${ }^{(2-3)}$.

A miopia aumenta o risco de DR, além de favorecer o aparecimento precoce da lesão, por causa direta - pela deficiência da matriz que une os fotorreceptores ao epitélio pigmentado retiniano e/ou pela alteração do mecanismo de bomba do epitélio pigmentado retiniano - ou por causa indireta - pelo estiramento da retina, deixando-a suscetível a lesões degenerativas e roturas causadas por uma menor resistência à tração vítreoretiniana ${ }^{(4-6)}$. O DR está associado às degenerações periféricas da retina as quais são identificadas por meio da observação clínica, podendo ser classificadas pela morfologia, prognóstico e predisposição ou não ao $\mathrm{DR}^{(7)}$.

A cirurgia refrativa realizada em grande escala é um procedimento recente. Mais recente ainda é a técnica laser in situ keratomileusis (LASIK) que vem se popularizando desde a segunda metade da década passada até hoje. Portanto é escassa a informação existente no que tange à injúria da retina após o LASIK, existindo poucos relatos na literatura que atribuem ao LASIK causa de DR. Como o número de míopes que se submetem ao LASIK é cada vez maior, é natural que entre esses pacientes estejam incluídos aqueles predispostos ao DR, bem como à hemorragia macular e às outras doenças vítreo-retinianas, sendo compreensível que essas anormalidades, e/ou suas exacerbações, sejam associadas ao próprio LASIK ${ }^{(8)}$.

O DR afeta uma pequena percentagem da população geral, dependendo da presença ou ausência de degenerações periféricas retinianas. Na população geral, ocorre na proporção de 1:10.000 por ano. Aproximadamente 40 a 
55\% dos DR afetam pacientes míopes, sendo que a incidência de DR em míopes é de 1 a 3\% ${ }^{(3,9)}$. Ogawa \& Tanaka analisaram 1.116 casos de DR e encontraram miopia em $82,16 \%$ deles $^{(10)}$. Estatisticamente, olhos míopes apresentam probabilidade de ter DR em 2,4\% dos casos, enquanto emétropes $0,06 \%$, ou seja, 40 vezes menos. Existe também uma relação entre a gravidade da miopia e a frequiência de $\mathrm{DR}^{(11)}$. Quando se comparam pacientes míopes, verifica-se que o descolamento regmatogênico de retina é de três a cinco vezes mais freqüente nos míopes e quinze vezes mais freqüente nos portadores de alta miopia. Um olho portador de erro refracional com equivalente esférico entre $-1,00$ e -3,00 D apresenta risco de DR quatro vezes maior que um olho não míope; em casos onde o erro refrativo é maior que $-3,00 \mathrm{D}, \mathrm{o}$ risco seria dez vezes maior. Em míopes com erro refracional superior a -15,00D, a frequiência de DR é 68,6 vezes maior que para o médio hipermétrope ${ }^{(10)}$. A maioria dos míopes tem o comprimento axial aumentado, causando anormalidades vítreas e periféricas na retina, que são os principais fatores predisponentes ao DR. Como o número de míopes que se submetem ao LASIK é cada vez maior e pela predisposição natural dos míopes ao DR, é de se esperar que, com a cirurgia, os casos de DR aumentem. Porém para que haja o DR, é preciso que existam alterações na anatomia normal da retina, como o aparecimento de degenerações na periferia e no fundo do olho. Atualmente, a grande maioria dos cirurgiões refrativos se preocupa em realizar exames de fundoscopia para identificar possíveis alterações periféricas da retina e quando necessário tratá-las profilaticamente. Porém, existem controvérsias sobre quais degenerações necessitam de tratamento profilático ${ }^{(8,12)}$, sendo este o objetivo desta revisão bibliográfica.

Embora alguns estudos ${ }^{(13)}$ mostrem casos de DR após o LASIK, publicamos recentemente um estudo prospectivo, realizado na Universidade de Brasília, que demonstrou não haver relação de causa e efeito entre o erro refrativo, o procedimento corretivo e as complicações retinianas ${ }^{(8)}$. A associação entre patologia retiniana e LASIK prévio parece refletir apenas a predisposição natural dos míopes a essas complicações. Sugere-se então que qualquer lesão retiniana que predisponha o olho ao desenvolvimento de DR seja profilaticamente tratada antes da cirurgia refrativa ${ }^{(14-17)}$.

Atualmente o LASIK se tornou a opção mais popular para a correção de baixas e moderadas ametropias. Nos Estados Unidos, o número atual de procedimentos de LASIK realizados por ano está entre 900.000 e 1,1 milhão. Na Europa, a estimativa do mercado total para o LASIK é de mais de 1 milhão de procedimentos por ano. No Japão, onde o LASIK aos poucos está ganhando popularidade, foram executados 8.000 procedimentos em 1999. Calcula-se que na América Latina o LASIK seja realizado em aproximadamente 300 mil pacientes por ano, sendo a maioria míopes entre - $0,50 \mathrm{D}$ e -10,00 D. A prevalência da miopia nos Estados Unidos varia de $25 \%$ a $46,4 \%$ na população adulta. Nas populações asiáticas a proporção da miopia pode ser muito mais alta; no continente africano e nas ilhas do Pacífico a incidência da miopia é bem mais baixa. Atualmente, a cirurgia refrativa a laser é a mais indicada para pessoas com baixa miopia (menos de $-5,00 \mathrm{D}$ ). Nos casos de miopia moderada (-5,01 a -10,00 D) e dependendo da espessura da córnea, também existe boa indicação para cirurgia a laser. A maioria dos pacientes se enquadra em um desses dois grupos. Nos pacientes com graus mais elevados de miopia as técnicas mais indicadas têm sido o implante de lente intra-ocular em olho fácico ${ }^{(11)}$.

Cinco a 10\% dos casos de DR, após tratamento cirúrgico, apresentam complicações pós-operatórias variáveis. Do total de casos com êxito anatômico pós-operatório, cerca de $40 \%$ têm acuidade visual final melhor que $6 / 15^{(18)}$. Assim, em função das graves consequiências do DR, tanto sob o ponto de vista anatômico quanto funcional, os esforços devem se concentrar na profilaxia e no tratamento precoce antes do acometimento macular ${ }^{(19)}$.

A prevenção do DR pode ser feita de três formas: evitando a liquefação vítrea e o descolamento posterior do corpo vítreo (DVP); aliviando a tração vítreo-retiniana e localizando e tratando, de acordo com critério de risco, as lesões retinianas com potencial para causar descontinuidades teciduais da retina, precursoras do descolamento regmatogênico de retina ${ }^{(19)}$. No entanto, não há como evitar a liquefação vítrea, visto que ela é parte das alterações que ocorrem com o envelhecimento ${ }^{(5,20)}$. As trações vítreo-retinianas, pelo risco de complicações e pela impossibilidade técnica de (no caso da vitrectomia) retirar o vítreo anterior-periférico nos olhos fácicos (sendo este o principal fator na formação de novas roturas), são aliviadas apenas em casos específicos, onde existam tração e alto risco de $\mathrm{DR}^{(21)}$.

As degenerações periféricas da retina podem aparecer durante o desenvolvimento ocular. Nos casos em que o olho apresenta crescimento maior que o normal, a retina teria dificuldade para recobri-lo internamente de forma adequada, ou seja, existe mais área a ser ocupada pela retina do que a sua própria área; com isso ocorre um afinamento do tecido retiniano na tentativa de ocupar toda a área interna do olho. Outra teoria para o aparecimento dessas degenerações é a tração que o corpo vítreo exerce sobre a retina quando dela se descola, havendo então um esgarçamento da retina com o conseqüente aparecimento das degenerações ${ }^{(8)}$.

O risco mais grave para o olho que apresenta degeneração periférica da retina é o aparecimento de buraco retiniano, o que pode causar o desprendimento da retina de sua posição original $^{(8,22-23)}$.

Entre as degenerações mais graves, destaca-se a degeneração em paliçada (degeneração lattice), o branco sem pressão, os microburacos retinianos e os buracos propriamente $\operatorname{ditos}^{(24)}$.

As degenerações periféricas da retina são causadas pelo enfraquecimento de certas áreas do tapete retiniano e acometem $70 \%$ dos olhos míopes. Essa fragilidade pode ser dividida em dois grupos: aquele onde há gravidade e o prognóstico é reservado $(10,24 \%)$ e outro com bom prognóstico sem riscos futuros $(59,76 \%)^{(25-27)}$.

Bec et al. ${ }^{(28)}$ sugeriram uma classificação dependendo do tecido acometido (retina, coróide ou corpo vítreo): 
- Lesões degenerativas intra-retinianas:

- Degeneração microcistóide típica

- Degeneração microcistóide reticular

- Retinosquise senil

- Cistos na pars plana

- Lesões degenerativas vítreo-retinianas:

- Degeneração em paliçada (lattice)

- Degeneração em rastro de lesma (snail track)

- Degeneração do tipo branco com pressão e branco sem pressão

- Lesões degenerativas coriorretinianas:

- Degeneração pavimentosa (pavingstone)

- Distúrbio pigmentar periférico

- Disseminação pigmentar difusa: degeneração orocoroidal, drusas e favo de mel (honeycomb)

\section{Lesões degenerativas intra-retinianas}

A degeneração microcistóide é a mais freqüente da periferia da retina ${ }^{(3,28)}$, sendo que a freqüência desse tipo de degeneração aumenta com a idade, chegando a $100 \%$ dos examinados quando se usam técnicas de exame mais apuradas ${ }^{(20,29-30)}$. Podem ocorrer em duas formas: típica, que consiste em espaços cistóides nas camadas médias da retina, envolvendo a camada plexiforme externa e a forma reticular, que envolve as camadas internas, como a camada de fibras nervosas da retina. Esse tipo de degeneração não oferece riscos para a retina, podendo estar associada à retinosquise e só excepcionalmente ocorrendo a formação de buracos retinianos, o que exigiria tratamento profilático $^{(20,30-31)}$.

A retinosquise não é um verdadeiro cisto; ela produz a separação de duas camadas internas da retina sendo mais comum após os 40 anos. Não há predileção por sexo e atinge 2 a 4\% da população. Essa lesão se apresenta como uma elevação bolhosa da retina periférica mais comumente no quadrante temporal inferior, sendo freqüentemente bilateral e simétrica ${ }^{(28,32-35)}$.

Um tipo de lesão considerada predisponente ao DR é o tufo cístico de retina. Trata-se de um remanescente embriológico caracterizando-se por uma projeção de tecido retiniano, branco-acinzentado, de aproximadamente $1 / 4$ de diâmetro do disco óptico, anterior ao equador do olho, próximo a ora serrata, com traves vítreas aderidas em seu ápice tornando-as potencialmente predisponentes para o desenvolvimento de roturas por tração vítrea e $\mathrm{DR}^{(8)}$. Cistos da pars plana são encontrados em 5 a $10 \%$ da população e apresentam-se como uma bola pequena e transparente sobre a ora $\operatorname{serrata}^{(36-38)}$. Trata-se de uma clivagem entre o epitélio pigmentado e o epitélio não-pigmentado da pars plana. Sua freqüência é similar nos dois sexos, e por ser uma lesão inócua não precisa ser $\operatorname{tratada}^{(28)}$.

Devemos recordar que, apesar de o tufo cístico de retina estar em grande parte relacionado aos buracos com opérculos livres, são os cistos da pars plana mais relacionados às roturas em ferradura que levam ao descolamento regmatogênico de retina ${ }^{(37)}$. No grupo dos altos míopes essa degeneração é mais preocupante, pois é neste grupo que cirurgias de miopia podem induzir ao $\mathrm{DVP}^{(38-39)}$.

\section{Lesões degenerativas vítreo-retinianas}

A degeneração lattice ou em paliçada, ocorre em cerca de 8 a $20 \%$ da população, sendo mais comum nos míopes ${ }^{(28,40-42)}$. A incidência de degeneração em paliçada é de $22 \%$ nos emétropes, $37 \%$ nos míopes entre 0 e 3 dioptrias, $26 \%$ nos míopes entre 3 e 8 dioptrias e $15 \%$ nos míopes com mais de 8 dioptrias. A incidência dessa lesão é máxima na segunda década de vida, sendo que novas lesões podem ocorrer em apenas $0,25 \%$ dos casos após os $19 \operatorname{anos}^{(40)}$. É freqüentemente bilateral e simétrica e tem maior freqüência no quadrante temporal superior ${ }^{(28)}$. Trata-se da degeneração retiniana mais freqüentemente relacionada aos casos de descolamento de retina ${ }^{(43)}$. A degeneração lattice é área de afinamento da retina com forma fusiforme e alterações pigmentares bem demarcadas e acometendo mais comumente a região entre o equador e a base vítrea. Apresenta vasos obliterados e fibrosados em rede e promove a sinérese (liquefação vítrea). Muitas vezes está acompanhada das degenerações em flocos de neve e branco com pressão, evoluindo para roturas em $2 \%$ e buracos em $30 \%$ dos acometi$\operatorname{dos}^{(28,44)}$. Parece existir uma relação inversamente proporcional entre o grau de miopia e a presença de degeneração em paliçada, isto é, quanto maior o equivalente esférico, menor a prevalência da lesão(45-46). A presença de degeneração em paliçada aumenta a possibilidade de descolamento regmatogênico de retina em dez vezes ${ }^{(27)}$. Um indivíduo portador de alta miopia e com essa degeneração apresenta 150 a 200 vezes mais chances de desenvolver DR do que um indivíduo nãomíope ${ }^{(47)}$. Em pesquisa realizada nos EUA, $17 \%$ dos cirurgiões fazem tratamento profilático nos casos de degeneração lattice quando estas são diagnosticadas e 4\% dos cirurgiões só fazem este tratamento profilático nos casos de degeneração lattice associada a buracos retinianos antes de facectomia ${ }^{(8)}$. Já a escola francesa preconiza tratamento profilático com fotocoagulação a laser em $360^{\circ}$ no caso de diagnóstico da degeneração lattice $e^{(8,28)}$.

A degeneração em rastro de lesma (snail track), descrita por Gonin em 1904, ocorre com mais freqüência nos jovens e míopes não aumentando com o passar dos anos ${ }^{(28)}$. Consiste numa degeneração dos elementos neurais com depósito de lipídios nas camadas internas da retina; para alguns autores, ela é uma precursora da degeneração lattice. Apresenta-se sob a forma de pontos brancos agrupados em áreas, formando placas ou bandas. Como favorece o desenvolvimento de buracos retinianos, este tipo de lesão deve ser tratada profilaticamente $\mathrm{e}^{(15,41,48)}$

As degenerações do tipo branco com pressão e branco sem pressão, descritas primeiramente por Rutnin \& Schepens $^{(36)}$, acometem os dois sexos igualmente, existindo uma relação entre esta degeneração e a prematuridade ${ }^{(28)}$. A degeneração do tipo branco com pressão tem aspecto branco acinzentado induzido pela denteação escleral ${ }^{(49)}$. Trata-se de 
uma degeneração benigna sem relação com o DR e muda de aspecto de acordo com a mudança da identação escleral. A degeneração do tipo branco sem pressão (white without pressure - WWP) apresenta-se como a anterior, porém sem a identação escleral. Na maioria dos pacientes esta degeneração é inócua, porém pode se desenvolver e levar ao DR. As degenerações do tipo branco com e sem pressão representam um conjunto de alterações gerais inespecíficas ${ }^{(8)}$. Estudos mostram que, nas miopias moderada e alta, as degenerações do tipo branco sem pressão e branco com pressão apresentam respectivamente a incidência de 2,3 e 3,5 vezes maior que na miopia baixa, o que confirma que a freqüência dessas alterações está diretamente relacionada ao grau de miopia ${ }^{(3)}$. Alguns autores defendem o tratamento profilático dessas lesões, porém atualmente esta é uma conduta bastante questionada pelos retinólogos. A presença do branco com e sem pressão não influi no risco de DR, a não ser no olho contralateral de pacientes com rotura gigante; neste caso específico, justifica-se o tratamento profilático ${ }^{(8)}$.

\section{Lesões degenerativas coriorretinianas}

A degeneração pavimentosa (pavingstone), descrita por Donders há cem anos, é uma atrofia do tecido retiniano em áreas circunscritas, com área de um diâmetro do disco óptico, simulando um calçamento de rua ${ }^{(28)}$. Histologicamente tratase de um afinamento da retina com ausência do epitélio pigmentado $^{(50)}$. É encontrada na freqüência de $15 \%$, sendo que $38 \%$ dos casos são bilaterais, não havendo preferência por $\operatorname{sexo}^{(51)}$. A freqüência dessa alteração está diretamente relacionada ao grau de miopia, pois essa alteração é 2,2 e 4,3 vezes mais freqüiente nas miopias moderada e alta, respectivamente $\mathrm{e}^{(3)}$. O quadrante temporal inferior é o mais acometido, com cerca de 78,5\% dos casos. Esse tipo de degeneração não apresenta relação com DR, portanto não oferece riscos para o paciente, tratando-se de um achado casual no exame de fundo de olho ${ }^{(52)}$.

A disseminação pigmentar difusa é uma degeneração natural do envelhecimento e ocorre em $10 \%$ dos pacientes, sendo que a frequiência nos míopes pode chegar a 50\%. São faixas pigmentadas e uniformes que ocorrem na periferia da retina, assim como a degeneração em favos de mel, que recebe este nome pela aparência característica. São degenerações inócuas que não necessitam de tratamento profilático ${ }^{(9)}$.

As drusas são degenerações pigmentares que acometem principalmente pacientes acima de 50 anos de idade. São pontos branco-amarelados que não trazem riscos para a retina no que se refere a um possível descolamento ${ }^{(53)}$.

A degeneração orocoroidal é um tipo de degeneração retiniana que se caracteriza por uma despigmentação difusa da coróide com um adelgaçamento da retina subjacente, atingindo todas as camadas retinianas ${ }^{(28)}$. A degeneração orocoroidal pode se estender da ora serrata até o equador, ocorrendo em cerca de $69 \%$ dos pacientes, sendo mais comum após os 40 anos de idade ${ }^{(8,28)}$. Apesar de ser considerada inócua pode originar o DR nos casos mais graves. Mas o tratamento profilático não é preconizado como regra geral ${ }^{(41)}$.

\section{Roturas e buracos retinianos}

Os buracos retinianos são lesões redondas, atróficas, bordas lisas, sem opérculo retiniano e sem opérculo vítreo. Apresentam mínima gliose e podem aparecer sem distúrbio do corpo vítreo. Acometem 2 a $7 \%$ da população geral e quase sempre são unilaterais e periféricos. Já as roturas retinianas podem ser divididas em totais ou parciais. As roturas parciais geralmente em forma de U ou V resultam do DVP; podem estar associadas a hemorragias vítreas ou retinianas, mas geralmente não causam descolamento de retina ${ }^{(42,54)}$. A rotura total é uma solução de continuidade da retina sensorial associada a opérculo retiniano e opérculo vítreo. É secundária à tração vítreo-retiniana e apresenta esclerose central e hiperplasia do epitélio pigmentado da retina. Acomete mais freqüentemente o hemisfério temporal (superiormente, em 35\% dos casos e inferiormente, em $32 \%$ ) na população geral, mais frequientemente os homens e na maioria das vezes é bilateral ${ }^{(28)}$.

A diálise retiniana é uma grande rotura na ora serrata, maior que $90^{\circ}$; recebe o nome de rotura gigante, podendo ser traumática ou espontânea. Apresenta duas linhas paralelas, uma branca e outra marrom, delimitando a rasgadura e mais freqüentemente atinge o quadrante temporal inferior ${ }^{(28)}$. A incidência de roturas varia com a idade, sendo maior na quarta, quinta e sexta décadas de vida, o que possibilita o desenvolvimento de roturas nos pacientes com o passar dos anos. Portanto, o acompanhamento desses pacientes no pós-operatório é importante, bem como a identificação de novas roturas e sua eventual relação com procedimentos cirúrgicos realizados ${ }^{(51,55-56)}$.

Byer em estudo com 223 olhos portadores de roturas retinianas e buracos, com e sem opérculo, de pacientes fácicos, assintomáticos e sem outra degeneração retiniana associada, observou que nenhum deles desenvolveu DR por aproximadamente sete anos e meio ${ }^{(23)}$.

Davis estudando 176 olhos não tratados, com roturas retinianas ou com descolamento de retina subclínico (definido como DR de um a dois diâmetros de papila em torno da rotura), referiu que $18 \%$ tiveram progressão para DR identificando três grupos em que ocorreu essa progressão: 1) olhos sintomáticos com roturas acima do meridiano horizontal; 2) olhos com roturas associadas ao descolamento de retina subclínico; 3 ) olhos afácicos com roturas, incluindo principalmente os que apresentavam descontinuidade retiniana antes da facectomia $^{(57)}$, A porcentagem de DR nesses grupos foi de $35 \%$, $30 \%$ e $50 \%$, respectivamente. Tais fatores podem estar presentes nos pacientes com indicação de cirurgia, principalmente nos altos míopes. $\mathrm{O}$ que justifica o tratamento profilático.

\section{Corpo vítreo}

O DVP ocorre de forma aguda, desprendendo o córtex do corpo vítreo da região foveal. Forma-se então um pertuito na hialóide por onde o corpo vítreo, que sofre um processo de sinérese, passa para o espaço sub-hialóideo. Mais comum nos míopes acima de $3 \mathrm{D}$, porém sem predileção por sexo ${ }^{(58)}$. $\mathrm{Na}$ 
maioria dos casos é assintomático, mas em 10\% dos casos ocorre uma aderência tão forte entre o vítreo e a retina, que, ao se descolar, o corpo vítreo traciona a retina que se descola. Portanto casos com sintomatologia (moscas volantes e flashes) e diagnóstico de DVP devem ser examinados com rigor. Caso haja defeito retiniano, este deve ser avaliado e tratado conforme a indicação ${ }^{(58-59)}$.

\section{Administração do paciente no pré-cirúrgico do LASIK}

Para que possamos fazer uma relação entre o LASIK e outra cirurgia ocular mais tradicional e estudada, vamos considerar a facectomia que é a cirurgia ocular mais freqüente no mundo. Barraquer et al. estudaram 165 olhos de 107 pacientes submetidos à extração do cristalino transparente e ao implante de lente intra-ocular para correção de alta miopia ${ }^{(47)}$. Encontraram 7,27\% dos olhos com DR, em média 30 meses após a cirurgia, sendo que destes, apenas $25 \%$ apresentaram DR nos primeiros seis meses. No mesmo estudo foi estimado que pacientes com menos de 30 anos tinham 2,5 vezes mais chances de descolamento regmatogênico de retina que os pacientes acima dessa idade e que aqueles submetidos à capsulotomia posterior apresentaram duas vezes mais DR que os com cápsula posterior intacta.

Assim, é possível que o DR, após cirurgias em pacientes com alta miopia, nas quais a câmara anterior é violada, seja causado por alterações fisiopatológicas secundárias à alta miopia, particularmente pela sinérese do corpo vítreo e não por uma ação direta da cirurgia. Freqüência semelhante foi encontrada em outros trabalhos, nos quais a incidência de DR, após facectomia em pacientes com alta miopia foi, respectivamente, de 3,5 e $9,6 \%{ }^{(59-60)}$.

É o grupo de alta miopia que causa maior preocupação ao oftalmologista, pois além das degenerações, as cirurgias de miopia podem induzir ou propiciar o aparecimento do $\mathrm{DVP}^{(61)}$.

Ao contrário das cirurgias que invadem a câmara anterior para correção de miopia, acredita-se que o DR nas cirurgias não-invasivas, como ceratotomia radial e fotoablação a laser, seja decorrente da história natural dos olhos míopes ou dos efeitos do uso de mióticos no pré e/ou pós-operatórios desses pacientes $^{(61-62)}$.

Outro fator importante que deve ser levado em consideração é a experiência do cirurgião de LASIK, pois a menor manipulação do olho diminui os fatores que levam ao DVP com possível tração retiniana ${ }^{(13,61-63)}$. Este seria um viés na avaliação da segurança do LASIK. Teorias como manuseio cirúrgico excessivo, descompressão aguda da câmara anterior pela sucção e influência dos efeitos das ondas do laser, questionam se o LASIK poderia aumentar a freqüência de lesões predisponentes ao DR. Acreditamos que estas teorias não procedem, como vem sendo demonstrado por algumas publicações literárias recentes. Porém lesões comprovadamente perigosas para a manutenção da anatomia habitual da retina, devem ser profilaticamente tratadas, prevenindo assim o aumento de incidência de $\mathrm{DR}^{(8)}$.
Também não menos importante é a sistemática da avaliação do segmento posterior do olho, que deve ser feito sempre com a atenção que exige um procedimento pré-cirúrgico. A avaliação das condições vítreo-retinianas pode ser fator preponderante para o sucesso do $\operatorname{LASIK}^{(11,17)}$.

O tratamento de escolha preconizado nas lesões predisponentes ao DR é a fotocoagulacão a laser, usando-se lente de três espelhos de Goldman ou quadrisférica, também podendo ser usado laser com oftalmoscopia binocular indireta em pacientes que não colaboram com o tratamento e necessitam de sedação. Recomenda-se que seja realizada a aplicação do laser de forma circunferencial à lesão $\left(360^{\circ}\right)$ em três filleiras distintas, sendo que a mais próxima da lesão deve guardar uma área de segurança das bordas da mesma, ou seja, o laser não deve ser aplicado sobre a lesão. Também se deve evitar aplicações de laser sobre o corpo ciliar. Os pontos de fotocoagulação devem ser eqüidistantes, alternando-se áreas semelhantes com e sem aplicação do mesmo. Em casos mais graves podemos fazer uso de crioterapia ou até mesmo uma cirurgia de identação com explante escleral ou vitrectomia via pars plana ${ }^{(8)}$.

Por fim não podemos esquecer que o olho operado para tratamento do erro refrativo, não é menos propenso aos defeitos retinianos, tão comuns nos míopes. Por isto a indicação de se realizar semestralmente a oftalmoscopia continua imperativa nestes olhos, principalmente para a visibilização da periferia retiniana ${ }^{(8,64-65)}$. O diâmetro da área de aplicação do laser na córnea em geral é de $6 \mathrm{~mm}$, o que não prejudica o exame do pólo posterior dos olhos que se submetem ao LASIK.

\section{ABSTRACT}

The purpose of this paper is to present the most frequent peripheral retinal degenerations in myopic eyes and discuss those degenerations which are a great risk for the retina and to point out the features associated with retinal detachment, its relation to LASIK and the indication for prophylactic treatment.

Keywords: Retinal degeneration/prevention \& control; Myopia/complications; Keratomileusis, laser in situ

\section{REFERÊNCIAS}

1. Waring GO. Myopia. In: Myopia: surgery. St. Louis: Mosby. 1992. p.4-15.

2. Ravault MP, Tressat C, Bertrand J. Degenerative lesions of the retinal periphery of the adelphic eye in patients with retinal detachment (clinical study). Bull Soc Ophtalmol Fr 1978;78:305-6.

3. Curtin BJ. The myopias: basic and clinical management. Philadelphia: Harper \& Row; 1995. p.337-9.

4. Karlin DB, Curton BJ. Peripheral chorioretinal lesions and axial length of the myopic eye. Am J Ophthalmol 1976;81:625-35.

5. Sebag J. Ageing of the vitreous. Eye 1987;1:254-62.

6. Austin KL, Palmer JR, Seddon JM, Glynn RJ, Rosenberg L, Gragoudas ES et al. Case-control study of idiopathic retinal detachment. Int J Epidemiol 1990;19:1045-50.

7. Kansky JJ. Peripheral retinal degenerations. Trans Ophthalmol Soc UK 1975; 95:173-9.

8. Nassaralla JJ. Estudo das alterações da retina periférica de pacientes portadores 
de miopia após cirurgia refrativa [tese]. Brasília: Universidade de Brasília; 2002

9. Benson WE, Morse PH. The prognosis of retinal detachment due to lattice degeneration. Ann Ophthalmol 1978;10:1197-200.

10. Ogawa A, Tanaka M. The relationship between refractive errors and retinal detachment - analysis of 1,166 retinal detachment cases. Jpn J Ophthalmol 1988;32:310-5.

11. Arevalo JF, Ramirez E, Suarez E, Cortez R, Antzoulatos G, Morales-Stopello J, Ramirez G, Torres F, Gonzalez-Vivas R. Rhegmatogenous retinal detachment in myopic eyes after laser in situ keratomileusis. Frequency, characteristics, and mechanism. J Cataract Refract Surg 2001;27:674-80.

12. Nassaralla BA, Nassaralla JJ. Laser in situ keratomileusis after penetrating keratoplasty. J Refract Surg 2000;17:1-7.

13. Arevalo JF, Ramirez E, Suarez E, Morales-Stopello J, Cortez R, Ramirez G, Antzoulatos G, Tugues J, Rodriguez J. Fuenmayor-Rivera D. Incidence of vitreoretinal pathologic conditions within 24 months after laser in situ keratomileusis. Ophthalmology 2000;107:258-62.

14. Byer NE. The natural history of the retinopathies of retinal detachment and preventive treatment. Isr J Med Sci 1972; 8:1417-20.

15. Byer NE. Application of a new technique to a subtle diagnostic problem. Ophthalmology 2000;107:1211.

16. Byer NE. Can rhegmatogenous retinal detachment be prevented? Reflections on the history of "prophylactic" treatment of retinal detachment. Ophthalmologe 2000;97:696-702.

17. Wilkinson CP. Evidence-based analysis of prophylactic treatment of asymptomatic retinal breaks and lattice degeneration. Ophthalmology 2000;107:12-5.

18. Hilton GF, McLean EB, Chuang EL. Pathogenesis and natural history. In: HILTON, G.F., MCLEAN, E.B., BRINTON, D.A. Retinal detachment: principles and practice. $2^{\text {nd }}$ ed. San Francisco: American Academy of Ophthalmology; 1995. p.3-37.

19. Wilkinson CP, Rice, T.A. Michels retinal detachment. St. Louis: Mosby, 1997

20. Foos RY, Feeman SS. Reticular cystoid degeneration of the peripheral retina. Am J Ophthalmol 1970;69:392-403.

21. Freeman HM. Fellow eyes of giant retinal breaks. Trans Am Ophthalmol Soc 1978;6:343-82.

22. Byer NE. Clinical study of retinal breaks. Trans Am Acad Ophthalmol Otolaryngol 1967; 71:461-73.

23. Byer NE. The natural history of asymptomatic retinal breaks. Ophthalmology 1982;89:1033-9.

24. Byer NE. Prognosis of asymptomatic retinal breaks. Mod Probl Ophthalmol 1974;12:103-8.

25. Kumar J, Paul SD, Singh K. Periphery of the retina. A clinical study. Ophthalmologica 1971;163:150-70.

26. Kottow M. Peripheral retinal degenerations and breaks. Albrecht Von Graefes Arch Klin Exp Ophthalmol 1980;214:53-60.

27. Byer NE. Lattice degeneration of the retina. Surv Ophthalmol 1979;23:21348.

28. Bec P, Ravault M, Arné JL, Trepsat C. The fundus periphery. New York: Masson; 1980.

29. Daicker B. Anatomie und pathologie der menschlichen retinociliaren fundus peripherie. Basel: Karger; 1972.

30. Straatsma BR, Foss RY. Typical and reticular degenerative retinoschisis. Am J Ophthalmol 1973;75:551-75.

31. Paul H. Cystoid degeneration of the retinal periphery and Lang's folds. Ber Zusammenkunft Dtsch Ophthalmol Ges 1977;74:141-6.

32. Byer NE. Spontaneous regression of senile retinoschisis. Arch Ophthalmol 1972;88:207-9.

33. Byer NE. The natural history of senile retinoschisis. Trans Am Acad Ophthalmol Otolaryngol 1976;81:458-71.

34. Byer NE. The natural history of senile retinoschisis. Mod Probl Ophthalmol 1977;18:304-311.

35. Byer NE. Long-term natural history study of senile retinoschisis with implications for management. Ophthalmology 1986;93:1127-37.

36. Rutnin U, Schepens CL. Fundus appearance in the normal eyes. 3. Peripheral degenerations. Am J Ophthalmol 1967;64:1040-62.
37. Foos RY. Postoral peripheral retinal tears. Ann Ophthalmol 1974;679-87.

38. Byer NE. Relationship of cystic retinal tufts to retinal detachment. Dev Ophthalmol $1981 ; 2: 36-42$.

39. Byer NE. Cystic retinal tufts and their relationship to retinal detachment. Arch Ophthalmol 1981;99:1788-90.

40. Straatsma BR, Allen RA. Lattice degeneration of the retina. Trans Am Acad Ophthalmol Otolaryngol 1962;66:600-13.

41. Byer NE. A clinical definition of lattice degeneration of the retina and its variations. Mod Probl Ophthalmol 1975;15:58-67.

42. Byer NE. Long-term natural history of lattice degeneration of the retina. Ophthalmology 1989;96:1396-401. [Discussion, p. 1401-2].

43. CambiaggiI A, Ciurlo G, Trillo M. La degenerazione a graticciata della retina. Minerva Oftalmol 1973;152:53-65.

44. Byer NE. Prognosis of asymptomatic retinal breaks. Arch Ophthalmol 1974; 92:208-10.

45. Shiomi Y. Study of Lattice degeneration of the retina. Part 2. Clinical features of lattice degeneration of the retina. Nipón Ganka Gakkai Zasshi 1981;85:26975.

46. Celorio JM, Pruett RC. Prevalence of lattice degeneration and its relation to axial length in severe myopia. Am J Ophthalmol 1991;111:20-3.

47. Barraquer C, Cavelier C, Mejia LF. Incidence of retinal detachment following clear-lens extraction in myopic patients. Arch Ophthalmol 1994;112:336-9.

48. Byer NE. What happens to untreated asymptomatic retinal breaks, and are they affected by posterior vitreous detachment? Ophthalmology 1998;105: 1045-9; discussion p.1049-50

49. Tasman W, Jaeger EA. Duane's clinical ophthalmology. Vol. 1. Philadelphia: Lippincott-Raven; 1995.

50. Straatsma BR, Foos RY, Freman SS. Degenerative diseases of the peripheral retina. In: Duane, T.D. Clinical ophthalmology. Philadelphia: Harper \& Row; 1986.

51. Hyams SW, Neumann E. Peripheral retina in myopia. With particular reference to retinal breaks. Br J Ophthalmol 1969; 53:300-6.

52. O'Malley P, Allen RA, Straatsma BR, O'Malley CC. Paving stone degeneration of the retina. Arch Ophthalmol 1965; 73:169-82.

53. Kanski JJ. The classification and terminology of peripheral retinal degeneration. Mod Probl Ophthalmol 1975;15:103-14.

54. Byer NE. Changes in and prognosis of lattice degeneration of the retina. Trans Am Acad Ophthalmol Otolaryngol 1974;78:OP114-25.

55. Tolentino FI, Schepens CL, Freeman HM. Vitreoretinal disorders. Philadelphia: WB Saunders; 1976.

56. Foos R.Y, Wheeler NC. Vitreoretinal juncture. Synchysis senilis and posterior vitreous detachment. Ophthalmology 1982;89:1502-12.

57. Davis MD. The natural history of retinal breaks without detachment. Trans Am Ophthalmol Soc 1973;71:343-72.

58. Byer NE. Natural history of posterior vitreous detachment with early management as the premier line of defense against retinal detachment. Ophthalmology 1994;101:1503-13; discussion p.1513-4.

59. Coonan P, Fung WE.; Webster RGJr, Allen AWJr, Abbott RL. The incidence of retinal detachment following extracapsular cataract extraction. A ten-year study. Ophthalmology 1985;92:1096-101.

60. Lindstrom RL, Lindquist TD, Huldin J, Rubenstein JB. Retinal detachment in axial myopia following extracapsular cataract surgery. Trans New Orleans Acad Ophthalmol 1988; 36:253-68.

61. Luna JD, Artal MN, Reviglio VE, Pelizzari M, Diaz H, Juarez CP. Vitreoretinal alterations following laser in situ keratomileusis: clinical and experimental studies. Graefes Arch Clin Exp Ophthalmol 2001; 239:416-23. ok

62. Hofman RF, Starling JC, Hovland KR. Case report: retinal detachment after radial keratotomy surgery. J Refract Surg 1985;1:226.

63. Stulting RD, Carr JD, Thompson KP, Waring GO, Wiley WM, Walker JG, Complications of laser in situ keratomileusis for the correction of myopia. Ophthalmology 1999;106:13-20.

64. Nassaralla Junior JJ, Nassaralla BRA. Descolamento de retina após cirurgia refrativa. Rev Bras Oftalmol 1998;57:305-9.

65. Loewenstein A, Goldstein M, Lazar M. Retinal pathology occurring after excimer laser surgery or phakic intraocular lens implantation: evaluation of possible relationship. Surv Ophthalmol 2002;47:125-35. 